\title{
Indicador de Sustentabilidade Hídrica Urbana
}

\author{
Urban Water Sustentability Indicator
}

\section{Carlos Eduardo Morelli Tucci}

Submetido em: 08/02/17

Revisado em: 05/07/17

Aprovado em: 10/07/17
RESUMO

O ciclo sustentável da água ocorre quando a água usada retorna a natureza em quantidade e qualidade para ser utilizada por outros usuários, evitando comprometer estes recursos para as atuais e futuras geraçóes.

Este artigo analisa a sustentabilidade do ciclo de água urbano, considerando o uso doméstico da água. Da vazáo entregue a populaçáo, uma parte é consumida e uma parcela maior retorna para os rios. Quando o tratamento do esgoto doméstico éinadequado, os rios são poluídos, reduzindo a disponibilidade para jusante. Para avaliar estas condiçōes é proposto um indicador chamado de vazão de subsídio, definido como a vazão fornecida pelo ambiente para diluir a contaminaçáo gerada pela falta de tratamento de esgoto doméstico, entendida como um subsidio ambiental. Também é apresentado o Indicador de Sustentabilidade Hídrica, que relaciona a vazão utilizada pela populaçăo (consumo e diluição) e a vazão disponível.

Estes indicadores são determinados para o Brasil e analisados os cenários da melhoria dos índices de esgotamento sanitário e de eficiência do uso da água. Os resultados mostram que a vazão de subsídio é $9,0 \mathrm{~m}^{3} /$ habitante/dia, 27,9 vezes superior a vazão consuntiva. $\mathrm{O}$ indicador de sustentabilidade hídrica mostra que no cenário atual, a demanda de água no Brasil é 200 vezes a disponível, se considerarmos a disponibilidade da área onde a populaçáo está ocupando. Isto mostra o impacto que a população urbana produz na sua vizinhança quanto aos recursos hídricos.

Palavras-chave: Abastecimento de água; saneamento; sustentabilidade da água

\section{ABSTRACT}

Water sustainable cycle is the use of water and its return flow to nature in quantity and quality conditions to be used for others, avoiding to treat this resources for future generations.

This paper assesses the urban water sustainability cycle, considering de human consumption. From que amount delivered to population, the largest part return to the water bodies. When the sanitation services did not collect and treat the sewage, water bodies are polluted, diminishing the water availability. To assesses this conditions is proposed an indicator called "subsidy flow", defined as the flow required by the environment to recover the water body water quality by dilution. In addition, is presented the Water Sustainability Index which relates the used flow and the water yield.

These indicators were estimated for Brazil and assessed scenarios of improvement of sanitation services and water efficiency measures. The results showed a subsidy flow of $9 \mathrm{~m}^{3} /$ day/inhabitant, 27.9 times the consumptive flow. Water Sustainability Index is 200 for Brazil, which represents how many times of an urban area is required to supply the urban population.

Keywords: water supply; sanitation; water sustentability

\section{DESAFIOS DA SUSTENTABILIDADE HÍDRICA URBANA}

Os sistemas urbanos são primordialmente áreas de consumo e residências. São delimitados por áreas de alta densidade populacional e sustentado por sistemas biofísicos com área superior a urbanizada (REES, 2003). No início do século 20 a população urbana mundial era 13\% e para 2050 está previsto 69,6\% (UN, 2009). Esta evolução urbana aumentou a pressão sobre os recursos hídricos, produzindo impactos ambientais e reduzindo a qualidade de vida da população, na medida em que a mancha urbana se expande. 
Urbanização aumenta com o desenvolvimento econômico, já que emprego e renda mudaram da agricultura para serviços e indústria, junto com a melhoria de serviços para a populaçáo como educação, hospitais, centos de compra, residências e outros serviços. Grandes cidades se desenvolveram desde o século passado formando grandes manchas urbanas como São Paulo que tinha cerca de 200 mil habitantes no início do século vinte e terminou o século com 20 milhôes, representando um crescimento médio anual de 8,5\%. Em 2005 existiam 388 cidades maiores que 1 milhão de habitantes e 16 acima de 10 milhões e previsto que em 2010 haveriam pelo menos 60 cidades maiores de 5 milhóes (MCGRANAHAN e MARCOTULIO,2005).

$\mathrm{O}$ arranjo de uso da água e esgotamento sanitário das cidades é retirar água a montante das cidades nos rios, distribuir para a população e lançar seu efluente a jusante. $\mathrm{Na}$ bacia hidrográfica, a cidade recebe a poluição da cidade imediatamente a montante e transfere para a cidade a jusante o seu impacto, gerando um ciclo de contaminação ao longo da bacia (TUCCI,2007). A própria cidade se expande a montante dos mananciais contaminando suas fontes de água e reduzindo a disponibilidade hídrica em qualidade. Este processo perverso é resultado da falta de controle dos processos da urbanizaçáo da cidade, onde os principais impactos sobre o meio ambiente aquático são os seguintes: (a) falta de tratamento do efluente de esgoto doméstico e industrial; (b) Contaminação difusa do escoamento pluvial; (c) resíduos sólidos produzidos na cidade e devido a erosão urbana; (d) redução da recarga subterrânea devido a impermeabilização das superfícies urbanas; e (e) aumento das inundaçóes na drenagem urbana pelo aumento da frequência das vazóes e pela ocupação das áreas de risco de inundaçóes ribeirinhas.

A gestão integrada das águas urbanas (GIAU ou IUWM, sigla em inglês) visa reduzir estes impactos nas cidades na busca da sua sustentabilidade hídrica e ambiental. As medidas devem fazer parte dos serviços de saneamento básico: abastecimento de água, esgotamento sanitário, drenagem urbana e inundaçôes e resíduos sólidos, como prevê a legislação brasileira (BRASIL,2017). Estes serviços devem produzir soluçóes integradas, mas a realidade mostra que os serviços têm sido realizados de forma fragmentada, sem atender os princípios do GIAU.

Este artigo trata de introduzir índices de avaliação de sustentabilidade hídrica de áreas urbanas que cobrem parte dos impactos mencionados acima. Sáo apresentadas equaçóes genéricas e abordados principalmente indicadores relacionados com o uso da água e seu esgotamento sanitário.

\section{USO HUMANO E SEUS IMPACTOS NA QUALIDADE DA ÁGUA}

\section{Histórico das tendências do saneamento}

No início do século vinte a população urbana era pequena, mas já existiam metrópoles importantes como Londres, Paris, Nova York. A água era fornecida geralmente de fontes, como poços urbanos, que produziam frequentes epidemias de doenças transmitidas pela água. A água era contaminada pelo esgoto das pessoas, depositados em latrinas e poços.

A era higienista no início do século vinte teve como objetivo entregar água segura para a população e reduzir a mortalidade devido as doenças transmitidas pela água. Foi o início saneamento básico com foco no abastecimento de água segura. Todavia, os efluentes náo eram tratados e lançados sem tratamento na rede de drenagem (sistema de coleta misto das redes de cloacas) ou poços. O resultado foram rios urbanos contaminados, com impacto na qualidade de vida e no meio ambiente (TUCCI,2007).

O período corretivo ocorreu no início da década de 70, quando legislaçóes como o "clean water act" nos Estados Unidos estabeleceu que nenhum efluente deveria ser lançado nos rios sem o tratamento com a melhor tecnologia disponível. Foram realizados investimento subsidiados em tratamento de esgoto na Europa e Estados Unidos com recuperação dos rios.

No final dos anos 80, no Relatório Brundtland (de 1987) é apresentado o conceito de desenvolvimento sustentável ${ }^{1}$. Esta fase mudou a concepção dos sistemas urbanos a buscar a sustentabilidade fechando

\footnotetext{
1 "É o desenvolvimento que atende as necessidades do presente sem comprometer a habilidade das futuras gerações de obter suas próprias necessidades" - World Commission on Environment and Development, Our Common Future (1987).
} 
o ciclo com tratamento de todos os efluentes e preservação das condiçóes naturais de escoamento nas áreas urbanas, com prioridade para infiltração. Esta fase sustentável recebeu diferentes nomes como "Low Impact Development" (NAHB, 2004). Este nível foi atingido parcialmente por alguns países desenvolvidos e a ação envolve principalmente a redução das cargas difusas urbanas e rurais.

\section{Cenário do Brasil e de muitos países em desenvolvimento}

Parte importante dos países em desenvolvimento ainda está na fase higienista. Os rios estão permanentemente contaminados por falta de investimentos em coleta e tratamento de esgoto doméstico, mostrando indicadores deficientes e serviços inadequados. No Brasil, grande parte das empresas de Saneamento cobra o valor total dos serviços de esgoto quando coleta, mesmo sem tratar, portanto, qual o incentivo que terá para completar o serviço e buscar a sustentabilidade? A população está longe do problema por falta de educação e entendimento do problema e também pelo utilitarismo de náo pagar as contas. Observam-se redes de coleta que não tem ligação das casas, Estaçóes de Tratamento de Esgoto mal projetadas e operando de forma deficiente, e falta de objetivos de atendimento por parte dos prestadores de serviços e falta de fiscalização. O resultado desta ineficiência é que todos os rios urbanos estáo contaminados.

Em 1997 quando foi aprovada a lei de recursos hídricos no Brasil (BRASIL, 1997) o principal problema era falta de tratamento de esgoto, que perdura depois de 20 anos. $\mathrm{O}$ aumento de cobertura, principalmente de tratamento de efluentes, não acompanhou o crescimento da população e o desenvolvimento do país. A legislação de recursos hídricos introduziu a inovação do enquadramento dos rios com base no Conama 257/2005 que classifica o rio de acordo com a concentração de qualidade da água dos rios. O enquadramento (meta de qualidade da água) deve ser aprovado pelo comitê de bacia, mas necessita da concordância dos investidores que são as empresas de Saneamento que geralmente não atuam na direção de atingir as metas do enquadramento. $\mathrm{O}$ resultado das ineficiências institucionais e técnicas são os rios contaminados e foco de doenças.

\section{INDICADORES DE SANEAMENTO}

\section{SNIS - Sistema Nacional de Indicadores de Saneamento}

Os indicadores de saneamento raramente retratam as condiçôes sustentáveis dos sistemas hídricos. Estes indicadores mencionam a proporção de esgoto coletado e a proporção do esgoto tratado, mas raramente mencionam a meta final que é a qualidade da água dos corpos hídricos. Não existe conexáo entre as metas de saneamento e a qualidade de água do rio O SNIS (Ministério das Cidades) estabelece vários indicadores para os serviços de saneamento (SNIS, 2014). Na tabela 1 são destacados alguns destes indicadores utilizados. Estes indicadores estão relacionados com os serviços de saneamento e não sobre as consequências destes serviços sobre os sistemas aquáticos

Tabela 1 Alguns Indicadores do SNIS de saneamento

\begin{tabular}{c|c}
\hline $\begin{array}{c}\text { Indicadores } \\
\text { População total atendida com abastecimento de água (AG0019) (milhões } \\
\text { habitantes) }\end{array}$ & Valor \\
\hline $\begin{array}{c}\text { Volume de água produzido (AG006) milhões m }{ }^{3} \\
\text { Volume de água consumido (AG010) milhões de } \mathrm{m}^{3}\end{array}$ & $15.991,238$ \\
\hline $\begin{array}{c}\text { População atendida por esgotamento sanitário ES001) milhões } \\
\text { habitantes }\end{array}$ & $10.132,306$ \\
\hline Volume de esgoto coletado (ES005) milhões de $\mathrm{m}^{3}$ & 98,006 \\
\hline Volume de esgoto tratado (ES006) milhões de $\mathrm{m}^{3}$ & 5,357 \\
\hline
\end{tabular}




\section{Indicadores ambientais e na gestão de recursos hídricos}

A lei de recursos hídricos estabelece o enquadramento como uma meta de sustentabilidade para os rios. O enquadramento é aprovado pelo comitê de bacia e define qual a meta que a sociedade deseja para um determinado rio, em função dos usos e contaminaçôes na sua bacia. Este enquadramento é baseado nas classes dos rios definidos no CONAMA 357/2005 (CONAMA, 2005) e num conjunto de parâmetros de qualidade da água. São raros os rios urbanos que atendem as condiçóes de classe 2 (condiçóes ambientalmente aceitáveis dos rios).

Adicionalmente, não existe no país nenhuma regulação do sistema de meio ambiente que avalia ou estabelece classificaçấo ou mesmo enquadramento de rios considerando as cargas difusas

\section{INDICADOR DE VAZÃO DE SUBSÍDIO AMBIENTAL}

A demanda total de água é demanda consumida para uso urbano somada da demanda que o meio ambiente necessitaria para eliminar o efeito do esgoto não tratado, denominado aqui de vazão de subsídio ambiental. Este indicador tem um caráter educativo da falta de eficiência e do subsídio que cada pessoa está obtendo por não pagar pelos serviços adequados que feche o ciclo de sustentabilidade da água.

\section{Estimativa da vazão de subsídio ambiental}

Uma vazão q é entregue para consumo para a população. Este valor é geralmente expresso em L/dia/ pessoa. Desta vazáo q, retorna para os rios e ambientes aquáticos uma vazáo

$$
\mathrm{q}_{\mathrm{r}}=\propto . \mathrm{q}
$$

onde $\mathrm{q}_{\mathrm{r}}$ é a vazáo de retorno em $\mathrm{L} / \mathrm{dia} ; \propto$. é a proporção de vazão que retorna e não é consumida. Usualmente no abastecimento humano é adotado como sendo 0,8 .

A vazáo consumida fica sendo

$$
q_{c}=(1-\propto .) q
$$

Pelo conceito exposto para o indicador busca-se a vazáo necessária para diluir a carga do efluente do uso da água humano $\left(\mathrm{Q}_{\mathrm{r}}\right.$ ) que é $\mathrm{q}_{\mathrm{r}} \mathrm{C}_{\mathrm{r}}$ onde $\mathrm{C}_{\mathrm{r}}$ é a concentração da vazáo de retorno. A concentração resultante do rio $\left(\mathrm{C}_{s}\right)$ deve ser igual ou maior que as condiçóes estabelecidas para a qualidade do rio que possa ser novamente utilizada. Pode ser a concentraçáo do poluente analisado para a classe do rio $\left(\mathrm{C}_{\mathrm{s}}\right)$.

A equação de mistura é a seguinte

$$
C_{s}=\frac{q_{r} C_{r}+Q_{s} C_{i}}{q_{r}+Q_{s}}
$$

Onde $\mathrm{C}_{\mathrm{i}}$ é a concentração do corpo d'água em condiçōes naturais. O primeiro termo do lado direto representa a carga poluente e o segundo a carga de recuperação. Para um efluente coletado e tratado no nível esperado para o corpo receptor $Q_{s}=0$ e $C_{s}=C_{r}$.

A carga de retorno pode ser expressa pela seguinte expressão:

$$
\left.\mathrm{q}_{\mathrm{r}} \mathrm{C}_{\mathrm{r}}=\propto . \mathrm{q}\left[(1-\beta) C_{n}+\beta C_{T}\right)\right]
$$

Onde $\beta$ é a proporção de esgoto tratado da vazão de retorno $\mathrm{q}_{\mathrm{r}} ; \mathrm{C}_{\mathrm{n}}$ é a concentraçáo do esgoto não tratado; $\mathrm{C}_{\mathrm{T}}$ é a concentraçáo do efluente do esgoto tratado. Esta equaçáo pode ser desmembrada em vários termos que tenham níveis diferentes de concentração de efluentes.

Substituindo a equação 4 na equação 3 e isolando $\mathrm{Q}_{s}$ que é o indicador resulta na expressão seguinte

$$
Q_{s}=\frac{\propto \cdot q\left[(1-\beta) C_{n}+\beta C_{T}-C_{s}\right]}{\left(C_{s}-C_{i}\right)}
$$

Esta equação mostra que se desejarmos que $\mathrm{C}_{\mathrm{s}}=$ $\mathrm{C}_{\mathrm{i}}$, ou seja retornar as condiçôes naturais, será necessária uma vazão infinita $\left(\mathrm{Q}_{s}\right)$, portanto é necessário uma concentraçáo meta com valor superior (quando contamina) ao das condiçóes naturais. De outro lado se, houver $100 \%$ de coleta e tratamento $\left(\beta=1, \mathrm{C}_{\mathrm{T}}=\mathrm{C}_{\mathrm{s}}\right)$ a demanda para garantir a sustentabilidade é igual a zero $\left(\mathrm{Q}_{S}=0\right)$, situaçáo que fecha o ciclo da água.

Considerando que a Concentração do efluente tratado representa uma redução de concentração do esgoto "in natura", existirá uma relação onde 


$$
C_{T}=(1-\mu) C_{n}
$$

Onde $\mu$ é a proporção ( 0 a 1) da redução da concentraçáo e da carga. Considerando que ao construir uma ETE dificilmente a mesma terá uma redução de carga inferior a $50 \%$ e carga tratada dificilmente será $100 \%$, o valor de $\mu$ deve variar entre 0,5 e 0,95 .

Substituindo a equação 6 na equação 5 , resulta a seguinte expressão para a vazão sustentável

$$
Q_{s}=\frac{\propto \cdot q\left[(1-\mu \beta) C_{n}-C_{s}\right]}{\left(C_{s}-C_{i}\right)}
$$

Esta vazão é determinada em função da meta relacionada a um parâmetro de qualidade que caracteriza as condiçóes ambientais. Usualmente os indicadores são DBO, Demanda Bioquímica de Oxigênio, representativa da carga orgânica de efluentes domésticos. No caso de rios este é o principal indicador. No caso de lagos e reservatórios o fósforo, juntamente com o $\mathrm{DBO}$ são os indicadores principais.

\section{Estimativa com base no DBO}

Neste caso, espera-se para a classe 2 que o corpo d'água tenha até $5 \mathrm{mg} / \mathrm{L}\left(\mathrm{C}_{\mathrm{s}}\right)$. Um valor em condiçôes naturais espera-se que tenha da ordem de $2 \mathrm{mg} / \mathrm{L}\left(\mathrm{C}_{\mathrm{i}}\right)$.
A concentração do esgoto em natura é da ordem de $300 \mathrm{mg} / \mathrm{L}\left(\mathrm{C}_{\mathrm{n}}\right)$. O valor de $\propto .=0,8$. A demanda esperada é da ordem de $200 \mathrm{~L} /$ dia/habitante. A equação 7 fica a seguinte

$$
Q_{s}=\frac{160[(1-\mu \beta) 300-5]}{3}
$$

Para $\mathrm{Q}_{\mathrm{s}}$ em L/dia/habitante. Usando $\mathrm{m}^{3} / \mathrm{dia} / \mathrm{habi}$ tante a equação fica

$$
Q_{s}=16(1-\mu \beta)-0,26
$$

A equação fica dependente apenas da proporção de esgoto coletado e tratado e da reduçáo de carga. Caso $\mu \beta=1$ onde se tem todo esgoto coletado e redução de $100 \%$ da carga, a equação seria incoerente. Isto ocorre porque se aceita um nível de qualidade inferior a qualidade natural. Esta equação tem ajuste onde o maior valor de $\quad \mu=1-\frac{C_{s}}{C_{n}} \quad$ é nível de redução de carga equivalente a atender a concentraçáo meta do corpo hídrico.

$\mathrm{Na}$ figura 1 é apresentada a variação de $Q_{s} \mathrm{em}$ função de $\mu$ e $\beta$, ou seja, redução de carga e cobertura de coleta e tratamento. $O$ valor máximo de $Q_{s}$ é de $15,7 \mathrm{~m}^{3} / \mathrm{dia} /$ habitante quando os dois indicadores são nulos.

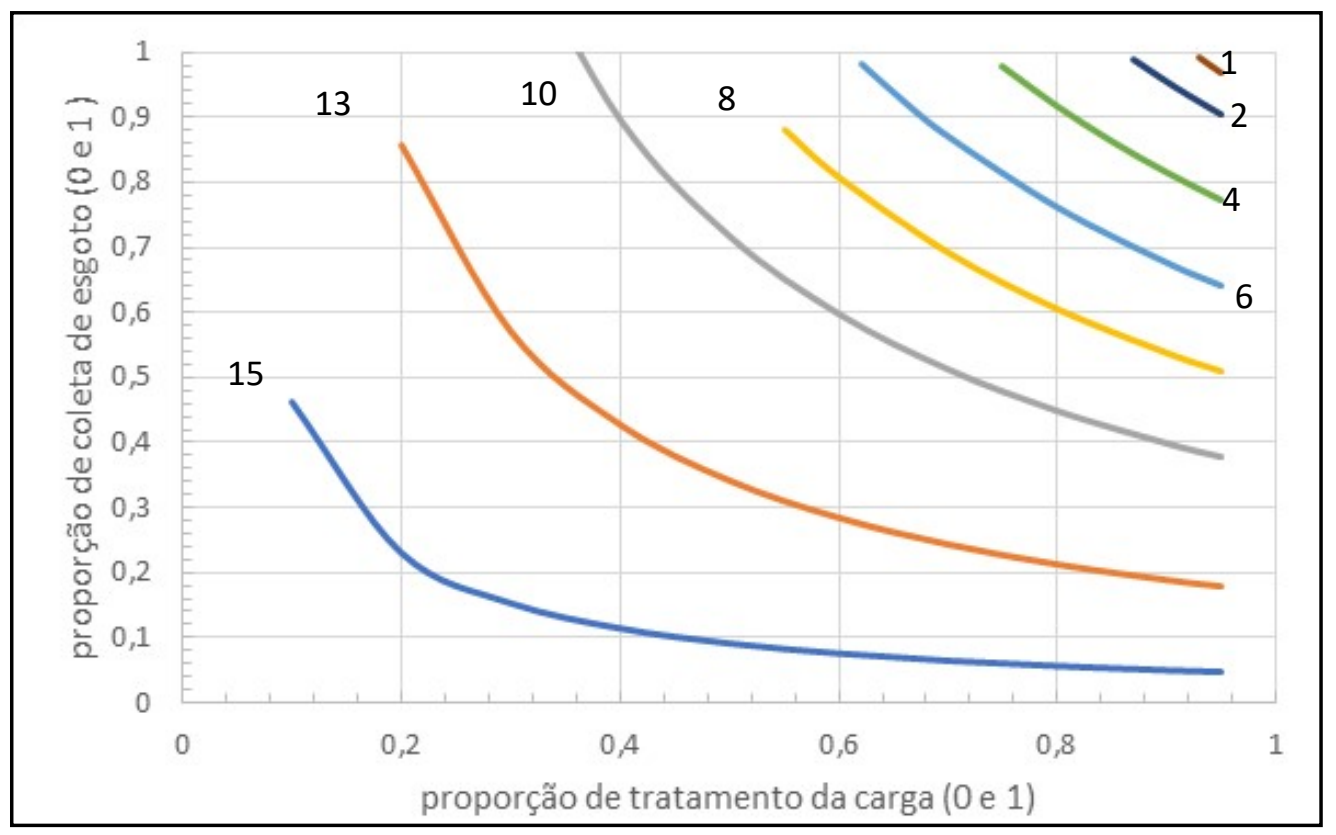

Figura 1 Valores Qs (m3/dia/pessoa) em função da redução de carga $(\mu)$ e cobertura de coleta e tratamento de esgoto $(\beta)$. O gráfico foi limitado a redução de carga de $95 \%$ 
Este também é o cenário se houver 100\% de coleta, mas sem tratamento, mostrando a ineficiência para o rio de existir apenas coleta. Com uma cobertura de $100 \%$ de coleta e tratamento de $85 \%$ da carga, que seria o esperado para uma meta adequada, resultaria em $\mathrm{Q}_{s}=2,1 \mathrm{~m}^{3} / \mathrm{dia} / \mathrm{hab}$. Para uma redução de carga de $95 \%$ o DBO resultaria numa demanda ainda de $0,5 \mathrm{~m}^{3} / \mathrm{dia} /$ habitante. Ainda muito superior a demanda consuntiva de $0,04 \mathrm{~m}^{3} / \mathrm{dia} /$ habitante. Este indicador mostra que a demanda de água para controle de qualidade é sempre superior a demanda de água consuntiva.

\section{Indicador com relação a vazão de consumo}

A equação 7 pode ser adimensionalizada em função da vazão de consumo. $\mathrm{O}$ resultado obtido $\left(\mathrm{q}_{s}\right)$ é várias vezes a vazão de consumo. A equação é a seguinte

$$
q_{s}=\frac{Q_{s}}{q_{c}}=\frac{\propto \cdot\left[(1-\mu \beta) C_{n}-C_{s}\right]}{(1-\alpha)\left(C_{s}-C_{i}\right)}
$$

Usando o valor de $\mathrm{C}_{\mathrm{n}}, \mathrm{C}_{\mathrm{s}}$ e $\mathrm{C}_{\mathrm{i}}$ adotados para $\mathrm{DBO}$ no item anterior resulta

$$
q_{s}=100(1-\mu \beta)-5 / 3
$$

Este indicador mede quantas vezes a demanda de qualidade da água é maior que a demanda consuntiva em função dos indicadores de cobertura e reduçáo da carga do esgoto bruto.

\section{Distribuição de acordo com a fonte de contaminação}

A carga não tratada é transferida para um rio por coletores de esgoto, enquanto que fossas e áreas de infiltração transferem a contaminação para os aquíferos. Em pequenas comunidades é comum o uso de fossa e a proporção de contaminação ocorre sobre o manancial subterrâneo. Nas cidades maiores onde existe economia de escala se reduz a cobertura de sistema com fossas e aumenta-se o sistema coletor e ETE.

Para identificar os mananciais contaminados quanto a distribuição de vazão de subsídio, introduziu-se nas equaçóes 7 e 11 um fator que caracteriza a parcela de uso de fossas e de rede coletora pluvial ou sanitária.
Nas primeiras a contaminação ocorre sobre as águas subterrâneas e na segunda a contaminação é sobre o sistema superficial.

Sendo $\mathrm{q}_{\mathrm{r}}$ a vazão de retorno haverá uma proporção que irá para o sistema de fossas $(\tau)$ e outra parcela para o sistema de coleta e tratamento de efluentes ou mesmo pluviais

A vazão subterrânea usualmente não é tratada, portanto não se aplicando os outros coeficientes e a vazão de diluição fica

$$
Q_{s}(\text { subt })=\frac{\tau \cdot \alpha \cdot q(C n-C s)}{(C s-C i)}
$$

A parcela que atinge o sistema aquático superficial é a seguinte

$$
Q_{s}(\text { superf })=\frac{(1-\tau) \propto \cdot q\left[(1-\mu \beta) C_{n}-C_{s}\right]}{\left(C_{s}-C_{i}\right)}
$$

Para uma determinada região ou bacia é possível identifica a quantidade de água que seria necessária para compatibilizar o subsídio tanto para os mananciais superficiais como os subterrâneos.

As equaçóes apresentadas foram apresentadas para uso unitário e pode ser multiplicado pela população para se obter os resultados para um universo desejado.

\section{MEDIDA RELATIVA DE SUSTENTABILIDADE}

As equaçóes apresentadas no item anterior retratam um cenário absoluto de demanda de água para cobrir a deficiência das ações da população, no entanto existem medidas relativas que podem ser examinadas em face dos limites quanto a Disponibilidade hídrica na regiấo ou bacia e risco de segurança hídrica;

\section{Limites da disponibilidade hídrica regional ou da bacia}

Considere uma regiáo ou bacia com uma disponibilidade hídrica $Q_{d}$. A disponibilidade hídrica é expressa por

$$
\mathrm{Q}_{\mathrm{d}}=\mathrm{k} \mathrm{Q}_{\mathrm{m}}
$$

Onde k é um fator inferior a 1 e $Q_{m}$ é a vazão média de longo período do local, uma variável de fácil estimativa. 
O fator $k$ depende da capacidade de regularização natural ou artificial existente na regiáo ou bacia. No Brasil, com exceção do semi-árido, o valor de k varia de 0,5 a 0,7 para reservatórios de regularização. Quando não existe reservatórios, a variável de disponibilidade hídrica usualmente adotada é a $Q_{55}$, neste caso $\mathrm{k}$ varia de 0,15 a 0,35 para as citadas regióes e a vazão específica média $\left(\mathrm{q}_{\mathrm{m}}\right)$ de 0,15 a $0,22 \mathrm{~L} / \mathrm{s} / \mathrm{km}^{2}$.

$$
\mathrm{Q}_{95}(\mathrm{~L} / \mathrm{s})=\mathrm{k} \cdot \mathrm{q}_{\mathrm{m}} \mathrm{A}\left(\mathrm{km}^{2}\right)
$$

Onde é a área da bacia em $\mathrm{km}^{2}$. Considerando que a demanda é obtida em $\mathrm{L} /$ dia, existe um fator de conversão que é $86400=86,4 \cdot 10^{3}$. Portanto, a equação 15 fica

$$
\mathrm{Q}_{\mathrm{d}}=\mathrm{Q}_{55}(\mathrm{~L} / \mathrm{dia})=86,4 \cdot 10^{3} \cdot \mathrm{k} \cdot \mathrm{q}_{\mathrm{m}} \cdot \mathrm{A}
$$

A demanda total da regiáo ou bacia para uso humano é

$$
\mathrm{Q}_{\mathrm{t}}=\mathrm{q}_{\mathrm{c}}+\mathrm{Q}_{\mathrm{s}}
$$

Neste caso, os valores estão multiplicados pela população e não representam valores unitários (equaçóes 2 e 7). A relaçáo entre a demanda total e disponibilidade expressa quanto dos recursos hídricos da região estáo comprometidos somente com a demanda da população urbana, ou seja

$$
\mathrm{IS}_{\mathrm{hi}}=\frac{Q_{t}}{Q_{d}}=\frac{q_{c}+Q_{s}}{Q_{d}}=\frac{\left\{(1-\alpha) q+\frac{\propto \cdot q\left[(1-\mu \beta) C_{n}-C_{s}\right]}{\left(C_{s}-C_{i}\right)}\right\} \cdot p}{Q_{d}}
$$

Onde ISI é o indicador de sustentabilidade hídrica da bacia ou da regiáo; p é a população da região ou bacia.

Reorganizando a equação fica

$$
\mathrm{IS}_{\mathrm{hi}}=\frac{q \cdot p}{Q_{d}\left(C_{s}-C_{i}\right)}\left\{(1-\alpha)\left(C_{s}-C_{i}\right)+\alpha\left[(1-\mu \beta) C_{n}-C_{s}\right]\right\}
$$

Esta equação permite estabelecer uma escala de criticidade de uma região ou bacia quando ao uso e impacto da qualidade da água e avaliar o limite de pessoas que podem ocupar uma regiáo de acordo com padróes de saneamento. Os recursos naturais estáo expressos em $\mathrm{Q}_{\mathrm{d}}$, o antrópico em $\mathrm{p}$ (população) e as ações de mitigação nos fatores de $\mu$ e $\beta$.

De outro lado a populaçáo ocupa um espaço que depende da sua densidade, expresso por

$$
p=A_{o c} \cdot D
$$

onde $\mathrm{A}_{\mathrm{oc}}$ é a área da mancha urbana em $\mathrm{km}^{2}$; $\mathrm{D}$ é a densidade da população $\left(\mathrm{hab} / \mathrm{km}^{2}\right)$. A densidade média da população no Brasil é de 65 hab/ha ou $6500 \mathrm{hab} / \mathrm{km}^{2}$ (Embrapa,2008), diminuindo para cidades menores e aumentando para cidades maiores. Em cidades muito densas da Asia e Africa chegam a valores superiores a $150 \mathrm{hab} / \mathrm{ha}$. Esta área é bastante inferior a área de disponibilidade hídrica. A relação entre as áreas pode ser expressa por

$$
\mathrm{f}_{\mathrm{a}}=\frac{A}{A_{o c}}
$$

Onde A é a área de drenagem $\left(\mathrm{km}^{2}\right)$ onde que engloba o manancial da região de ocupaçáo da população $\mathrm{p}$, ou de sua área de influência tanto como manancial como de recebimento do efluente. $\mathrm{O}$ valor mínimo de $\mathrm{f}_{\mathrm{a}}=1$ e o máximo depende envolve a dependência hídrica da mancha urbana.

Substituindo as expressóes 16, 20 e 21 na equação 19 resulta em

$$
\mathrm{IS}_{\mathrm{hi}}=\frac{\gamma}{f_{a}} \cdot\left\{(1-\alpha)\left(C_{s}-C_{i}\right)+\alpha\left[(1-\mu \beta) C_{n}-C_{s}\right]\right\}
$$

Onde $\gamma=\frac{q \cdot D}{86400 \cdot k \cdot q_{m}\left(C_{s}-C_{i}\right)} \quad$. O coeficiente foi introduzido para considerar as unidades das variáveis.

$\mathrm{O}$ valor de $\mathrm{IS}_{\mathrm{hi}}$ varia em função da densidade $\mathrm{D}$, demanda direta $\mathrm{q}$, da relação entre as áreas de ocupação e área de uso e impacto da água (bacia hidrográfica), das taxas de disponibilidade hídricas $\left(\mathrm{k}\right.$ e $\mathrm{q}_{\mathrm{m}}$ ), da meta de qualidade da água $\mathrm{C}_{\mathrm{s}}$ e dos indicadores de saneamento $\mu$ e $\beta$. 
O valor do indicador igual 1 mostra que o uso da água (quantidade e qualidade) é igual a disponibilidade. Quando este valor é superior a 1 o uso da água está em desequilíbrio com a disponibilidade e estará gerando impactos com qualidade da água em maior escala. De outro lado com o indicador inferior a 1 existe um equilíbrio global, mas ainda haverá potenciais problemas locais. Para equilibrar o indicador pode-se aumentar a área de disponibilidade e $\mathrm{f}_{\mathrm{a}}$ ou melhorar os índices de saneamento. No primeiro caso procura-se obter uma expansão da área de influência da área urbana que deveria ficar limitada a um espaço definido, enquanto no segundo busca-se um equilíbrio com os serviços de saneamento. Considerando $I_{\text {hi }}=1$ pode-se isolar a expressão em função de $f_{a}$, resultando em

$$
f_{a}=\gamma\left\{(1-\alpha)\left(C_{s}-C_{i}\right)+\alpha\left[(1-\mu \beta) C_{n}-C_{s}\right]\right\}
$$

No caso em que deseja-se conhecer o padrão de saneamento para uma proporção de área de ocupação com relação a área de manancial com o equilíbrio do índice de sustentabilidade hídrica resulta

$$
\mu \beta=\frac{\left(\frac{f a}{\gamma}-(1-\alpha)\left(C_{s}-C_{i}\right)+\gamma C_{s}\right)}{\gamma C_{n}}-1
$$

Utilizando os dados mencionados no item anterior para DBO onde $\mathrm{C}_{\mathrm{n}}=300 \mathrm{mg} / \mathrm{L} ; \mathrm{C}_{\mathrm{s}}=5 \mathrm{mg} / \mathrm{L} ; \mathrm{C}_{\mathrm{i}}=$ $2 \mathrm{mg} / \mathrm{L} ; \alpha=0,8, q=200 \mathrm{~L} / \mathrm{dia} /$ pessoa, é possível analisar a sustentabilidade de uma população de acordo com $\mathrm{f}_{\mathrm{a}}$.

Considerando $\mathrm{k}=0,2$ e $\mathrm{q}_{\mathrm{m}}$ (vazão específica média) $=20 \mathrm{~L} / \mathrm{s} / \mathrm{km}^{2}$. A densidade média do Brasil de 65 hab/ha. A equação 22 fica

$$
\mathrm{IS}_{\mathrm{HI}}\left(\mathrm{f}_{\mathrm{a}}, \mu, \beta\right)=\frac{1,254}{f_{a}}\{3 \alpha+(1-\alpha)[(1-\mu . \mathrm{B}) \cdot 300-5]\}
$$

$\mathrm{Na}$ figura 2 pode-se observar a relação entre as variáveis e a regiáo de valores maiores ou menores que 1 do indicador.

Com base nesta equação estima-se que para manter o equilíbrio do $\mathrm{IS}_{\mathrm{hi}}=1$, sem nenhuma coleta e tratamento de esgoto o valor de $\mathrm{f}_{\mathrm{a}}=296,6$ ou seja a área da disponibilidade hídrica é 296,6 vezes maior que $a$ área da mancha urbana. De outro lado se utiliza-se da melhor tecnologia disponível para coletar 100\% do esgoto e reduzir a carga para em $95 \% \mathrm{f}_{\mathrm{a}}=10,7 \mathrm{ou}$ a área da disponibilidade hídrica deve ser pelo menos 10,7 vezes a da área de ocupaçáo.

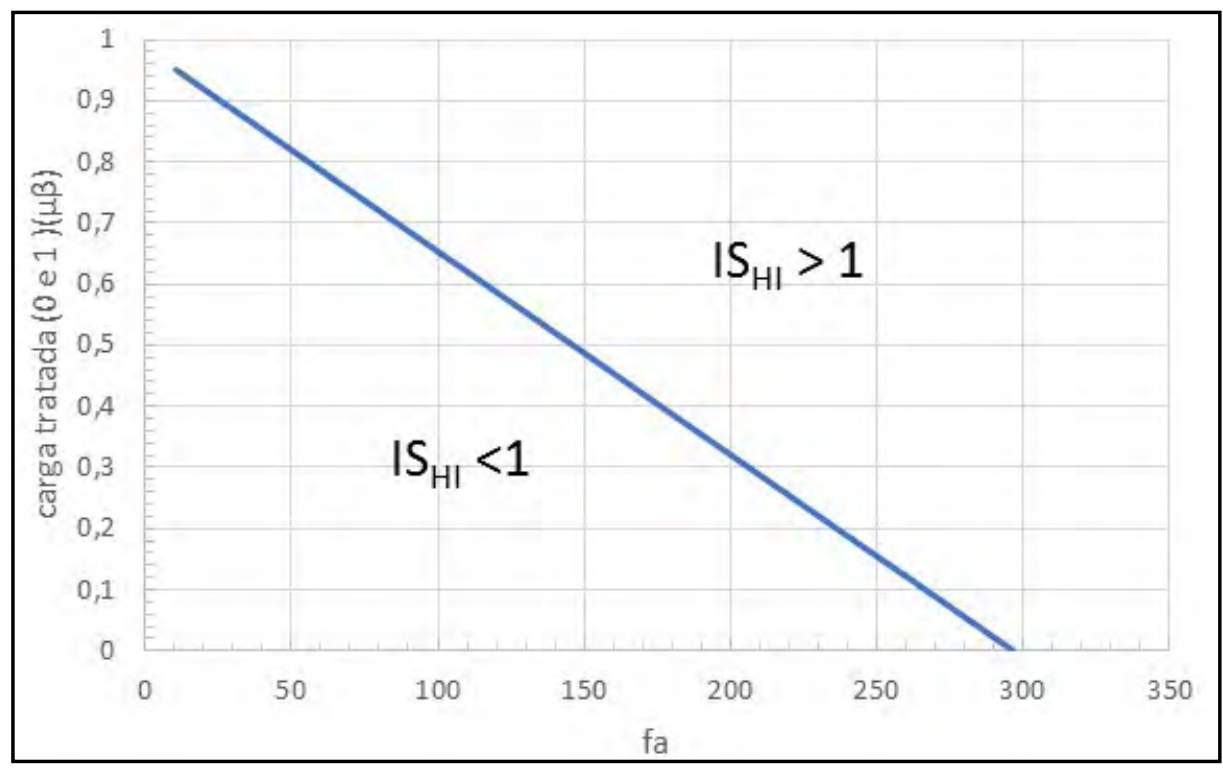

Figura 2 Variação do Indicador em função dos indicadores fa e carga tratada 
Para se buscar a sustentabilidade $\operatorname{com} f_{a}=1$ pode-se utilizar o seguinte: (a) reduzir o valor de $\alpha$ com reuso; (b) reduzir a demanda por pessoa q; (c) reduzir a densidade de ocupação. Alterando estes parâmetros para $\alpha=0,5$ e q $=160 \mathrm{~L} / \mathrm{dia} /$ hab e $\mathrm{D}=50 \mathrm{hab} / \mathrm{ha}$ e mantendo os níveis de cobertura de $100 \%$ e eficiência de $95 \%$ resulta para $\mathrm{fa}=5$ para $\mathrm{IS}_{\mathrm{Hi}}=1$, reduzindo o uso de recursos naturais em $50 \%$. Esta formulação permite uma avaliação integrada entre os indicadores dos mananciais e de conservação e eficiência dos sistemas de saneamento.

\section{ANÁLISE DOS INDICADORES}

A formulação apresentada para o indicador de sustentabilidade hídrica IS $_{\text {hi }}$ permite analisar a relação de influência entre a área urbana ocupada e a sua demanda por água e ou impacto $\left(\mathrm{f}_{\mathrm{a}}\right)$ para as áreas urbanas, utilizando os indicadores de saneamento e gestão das águas na cidade, medidas de eficiência, conservação e reuso para reduzir a expansão dos usos de recursos naturais em cada realidade.

Provavelmente apenas um indicador ou parâmetro de qualidade da água não será suficiente para avaliar todos os potenciais impactos para garantir a sustentabilidade.

O indicador apresentado não aborda outros impactos sobre as condiçóes hídricas como: erosão do solo, aumento da temperatura, contaminação das águas pluviais, mas é o primeiro passo para buscar de melhores indicadores que integrem o ambiente e o saneamento das cidades.

\section{RESULTADOS DOS INDICADORES}

\section{Uso da água no Brasil}

O uso da água no Brasil é sempre estimado com base na vazão direta utilizada pela cidade e calculado o seu valor por pessoa. Na tabela 2 são apresentados os principais indicadores de uso da água publicados pelo SNIS (2014). Na publicação os dados estão regionalizados por Estado, Regiáo, Prestadores de serviços, entre outros. Estas informaçóes foram obtidas com base na declaração dos prestadores de serviços cobrindo $98 \%$ da população brasileira. Considerando os indicadores da tabela 2, a demanda por pessoa é de $162 \times 0,20=$ $32,4 \mathrm{~L} / \mathrm{ha} /$ dia. Nestes indicadores a vazão entregue é de $162 \mathrm{~L} / \mathrm{ha} /$ dia e vazáo consuntiva de $32,4 \mathrm{~L} / \mathrm{ha} /$ dia.

\section{Vazão de Subsídio}

Considerando os indicadores da tabela 2, os parâmetros utilizados para determinar a equação de Q são apresentados na tabela 3, baseados nos indicadores brasileiros do SNIS. Neste caso, existe uma incógnita importante que é $\mu$, ou seja, o nível no qual são tratados os esgotos, em termos médios no Brasil. Um projeto de ETE geralmente trabalha com nível de reduçáo de $\mathrm{DBO}$ da ordem de $85 \%$, mas sabe-se que existem muitas estações com eficiência de projeto inferior e mesmo as projetadas, operando em nível fora do projeto. Portanto espera-se que o valor deste indicador varie entre 0,6 e 0,85. A concentração média do esgoto bruto pode variar muito de local para local e de região para regiáo em função da quantidade de escoamento pluvial no sistema de coleta. $\mathrm{O}$ valor utilizado como referência neste artigo é de $300 \mathrm{mg} / \mathrm{L}$.

Tabela 2 Indicadores selecionados de Água e Saneamento no Brasil (SNIS,2014)

\begin{tabular}{c|c}
\hline Indicador & Valor \\
\hline Perdas (\%) & 36,7 \\
\hline Consumo médio por pessoa L/habitante/dia & 162 \\
\hline Proporção de retorno para tratamento do volume (\%) & 80 a 85 \\
\hline Cobertura de coleta e tratamento' (\%) & 40,8 \\
\hline
\end{tabular}

1 - Não existem informações sobre a eficiência de tratamento do esgoto coletado 
O valor real de cada caso pode variar de 10 a $15 \%$ para cima ou para baixo. Os valores adotados para o Brasil são apresentados na tabela 3 abaixo.

Usando a equação 7 e os indicadores esperados obtidos na tabela 3 obtém-se que a vazão de subsídio no Brasil é de $9,042 \mathrm{~m}^{3} / \mathrm{dia} / \mathrm{hab}$ variando para os intervalos dos parâmetros entre 7,86 e $10,55 \mathrm{~m}^{3} / \mathrm{dia} /$ habitante. Somando-se a demanda consuntiva direta a demanda total que é a soma da vazão de subsídio e da demanda direta variam entre 7,89 e 10,58 com valor esperado de $9,07 \mathrm{~m}^{3} / \mathrm{dia} /$ habitante, onde a demanda de subsídio representa $99,7 \%$ da demanda total. Observa-se que a vazão consuntiva é insignificante perto da demanda total. A vazão de subsídio é 27,9 vezes a vazão consuntiva.

Com a melhoria do Saneamento para uma cobertura de $95 \%$ de coleta e $85 \%$ de eficiência de tratamento de esgoto ocorre uma redução da vazáo de subsídio para $2,28 \mathrm{~m}^{3} / \mathrm{hab} / \mathrm{dia}$

\section{Índice de Sustentabilidade Hídrica}

Cenário A - Para o cálculo do índice de sustentabilidade foram adotados valores de $\mathrm{k}=0,2$ e $\mathrm{qm}=17$ $\mathrm{L} / \mathrm{s} / \mathrm{km}^{2}$. Não foram realizadas variaçóes destes indicadores, apenas sobre os indicadores de saneamento, como no item anterior. $\mathrm{O}$ índice médio esperado para o Brasil é de 200,8 variando de 164,5 a 234,2. Observa-se que as áreas urbanas brasileiras estão usando da ordem de 200 vezes mais recursos hídricos que o existente na área que ocupa. Um índice bastante distante de uma sustentabilidade hídrica.

Cenário B - Para o cenário de cobertura quase total de saneamento, com aumento da cobertura de 40,8 para $95 \%$ de cobertura e uma eficiência aceitável de tratamento de esgoto de $85 \%$, o valor do índice cai para 51,1 , com redução de $74,5 \%$ do coeficiente.

Outros cenários: Outros fatores podem ser utilizados para reduzir ainda mais o impacto da cidade sobre os sistemas hídricos como: aumentar o reuso

Tabela 3 Indicadores utilizados para determinação da vazão de subsídio

\begin{tabular}{|c|c|c|}
\hline Indicador & Valor & Comentário \\
\hline $\mathrm{C}_{\mathrm{s}}(\mathrm{mg} / \mathrm{L})$ & 5 & $\begin{array}{l}\text { Considerando que classe } 2 \text { é a classificação adotada quando } \\
\text { não existe definição pelo comitê de bacia }\end{array}$ \\
\hline$C_{i}(\mathrm{mg} / \mathrm{L})$ & 2 & $\begin{array}{l}\text { Este valor pode variar de } 1 \text { a 3, mas é um valor aceitável para } \\
\text { esta consideração }\end{array}$ \\
\hline $\mathrm{Cn}(\mathrm{mg} / \mathrm{L})$ & 300 & Variando 10 a $15 \%$ com relação a este valor. \\
\hline$\alpha$ & 0,2 & $\begin{array}{l}\text { Varia de } 0,15 \text { a } 0,2 \text {. O valor adotado tem sido adotado pela ANA } \\
\qquad(A N A, x x) \text { no... }\end{array}$ \\
\hline$\beta$ & 0,408 & Valor do SNIS \\
\hline$\mu$ & 0,70 & $\begin{array}{c}\text { Varia de } 0,6 \text { a } 0,85 \text {. Valor adotado foi uma estimativa dentro } \\
\text { deste intervalo }\end{array}$ \\
\hline $\begin{array}{l}\text { q (consumo médio) (Litros/ } \\
\text { hab/dia) }\end{array}$ & 162 & Valor obtido do SNIS \\
\hline $\begin{array}{c}\mathrm{D} \\
\text { Densidade média (hab/km²) }\end{array}$ & 6500 & Este foi estimado para como valor médio para o Brasil \\
\hline K & 0,2 & $\begin{array}{c}\text { Valores variáveis de acordo com a geologia entre } 0,10 \text { e 0,35 } \\
\text { para regiões diferentes do semiárido }\end{array}$ \\
\hline qm & 17 & $\begin{array}{l}\text { Vazão específica média } \mathrm{L} / \mathrm{s} / \mathrm{km}^{2} \text {. Pode variar para regiões } \\
\text { diferentes do semiárido de } 12 \text { a } 30 \text {. O limite superior é a } \\
\text { Amazônia. }\end{array}$ \\
\hline
\end{tabular}


Tabela 4 Cenários de águas urbanas

\begin{tabular}{c|c|c|c|c}
\hline Cenários & Descrição & $\begin{array}{c}\mathrm{Q}_{\mathrm{s}} \\
\mathrm{m} 3 \text { habitante/dia }\end{array}$ & $\mathrm{IS}_{\mathrm{Hi}}$ & Redução' (\%) \\
\hline $\mathrm{A}$ & Atual & 9,042 & 200,8 & \\
\hline $\mathrm{B}$ & $\begin{array}{c}\text { Aumento de cobertura de saneamento para } \\
95 \%+85 \% \text { tratamento }\end{array}$ & 2,279 & 51,1 & 74,5 \\
\hline $\mathrm{C}$ & Redução de 10\% da demanda & 2,050 & 46,0 & 77,0 \\
\hline $\mathrm{D}$ & Cenário C +Aumento do reuso em 5\% & 1,922 & 43,4 & 78,4 \\
\hline $\mathrm{E}$ & Cenário D + aumento do tratamento para 90\% \\
da carga & 1,40 & 35,9 & 84,1 \\
\hline
\end{tabular}

1 redução com relação ao cenário $A$.

da água, reduzir a demanda e aprimorar os índices de saneamento. Um exercício sobre a variação destes fatores é apresentado na tabela abaixo, mostrando que o coeficiente pode chegar a 35,9 com redução de $84,9 \%$.

\section{Abrangência}

A população urbana brasileira segundo (SNIS,2014) é 178,378 milhôes e $26.365 \mathrm{~km}^{2} \mathrm{de}$ acordo com a densidade média de 65 hab/ha. No cenário a área urbana brasileira necessita 200 vezes a sua área urbana para compensar a falta de tratamento de esgoto, ou seja, $5.273 .300 \mathrm{~km}^{2}$. Este valor parece alto, mas considerando um sistema único, seria a área com disponibilidade hídrica necessária para diluir o esgoto náo tratado. A realidade mostra que isto não ocorre e a vizinhança das cidades é poluída.

\section{CONCLUSÕES}

O desenvolvimento econômico e social mundial tem sido primordialmente urbano desde o século passado. Este processo tem produzido uma concentração significativa de demanda de recursos naturais em espaços reduzidos, comprometendo a sustentabilidade da população. A demanda de recursos hídricos e os impactos gerados diretamente pelo uso humano da água é significativo. Atualmente a avaliação do uso dos recursos hídricos é limitada ao consumo da população, o que é uma falsa avaliação do efetivo impacto destas áreas, na medida que o efeito da falta de coleta e tratamento de efluentes náo é considerado.

Neste artigo foram introduzidos dois indicadores de sustentabilidade para avaliar e comparar os cenários de controle do uso da água nas cidades. O primeiro indicador foi denominado de "vazão de subsídio" que é o valor que a natureza necessitaria usar para diluir o efluente não tratado que retorna aos rios depois do uso da água pela população. Este valor deveria ser somado ao uso consuntivo da populaçáo para se ter o real valor da demanda de vazáo urbana. $\mathrm{O}$ segundo indicador foi denominado de índice de sustentabilidade hídrica que estima a relação entre a demanda de água e a sua disponibilidade num determinado local. Este valor estaria em equilíbrio se a área urbana usasse a água disponível na própria área, mas como isto não ocorre, este valor é muito superior a 1.

Estes indicadores foram estimados para as condiçôes médias do Brasil. Para isto usou-se os indicadores médios do país para interpretar o impacto do desenvolvimento urbano e seu uso da água humano sobre os recursos hídricos, em termos médios no país. Estimou-se que o Brasil tem uma demanda média de $9,04 \mathrm{~m}^{3} / \mathrm{hab} /$ dia e uma demanda consuntiva de $0,0324 \mathrm{~m}^{3} / \mathrm{hab} / \mathrm{dia}$, onde o primeiro representa $27,9 \mathrm{a}$ segundo. Este resultado mostra o significado impacto da falta de tratamento de efluente na demanda pela água no país, resultado da ineficiência dos serviços de saneamento. As equaçóes apresentadas mostram que este valor pode ser reduzido de forma significativa com base na ampliação dos serviços de saneamento.

$\mathrm{O}$ índice de sustentabilidade médio brasileiro chegou ao valor de 200, ou seja, a demanda de água é 200 vezes superior a oferta da sua própria área de ocupação. Outra interpretação é que são necessárias 200 áreas urbanas para fechar o balanço de demanda $\mathrm{x}$ disponibilidade, em termos médios, com os indicadores de saneamento atual. Este valor pode cair $80 \%$ com melhoria do saneamento e uso de medidas eficientes. 


\section{Referências}

BRASIL, 1997 Lei n.9433 de 8 de janeiro de 1997. Presidência da República do Brasil.

BRASIL, 2007 Lei n. 11.445 de 5 de janeiro de 2007. Presidência da República do Brasil.

BRASIL, 2016 Sistema Nacional de Informações sobre Saneamento: Diagnóstico dos Serviços de Água e Esgotos -2014 Ministério das Cidades Secretaria Nacional de Saneamento Ambiental SNSA 0212p

CONAMA,2005. Resolução 357 de 25 de março de 2005. Conselho Nacional de Meio Ambiente. Ministério do Meio Ambiente.

EMBRAPA, 2008 http://www.urbanizacao.cnpm.embrapa.br/conteudo/discussao.html. Acessado em 12/12/2008.

McGRANAHAN, G.; MARCOTULIO, P. 2005. Urban Systems. In: Ecosystem and Human Well-Being Current State and Trends. Volume 1. Island Press.

NAHB RESEARCH CENTER. 2004. Municipal Guide to Low Impact Development. Maryland. Disponível on-line em http://www.lowimpactdevelopment.org

REES, W.E., 2003. Understanding urban ecosystems: an ecologic economics perspective in: Understanding Urban Ecosystem: A new Frontier for Sciences and Education. A.R. Berkowitz, C.H. Nilon and K.S.Kollweg (eds) Sriger-Verlang, New York 115-136p.

TUCCI, C.E.M. 2007. Inundações Urbanas. ABRH Rhama. 380p (in portuguese).

UN,2009 Urban and Rural http://www.un.org/esa/population/publications/wup2007/2007urban rural.htm accessed in 01/16/2009.

Carlos E. Morelli Tucci Universidade Federal do Rio Grande do Sul e Rhama Consultoria Ltda, Porto Alegre, RS, Brasil

Contribuição do autor:

E-mail: tucci@rhama.com.br 